\title{
Adjuvant Therapy for Gastric Cancers: More Answers or More Questions?
}

\author{
Theodore S. Hong, $\mathrm{MD}^{1}$ and Eunice L. Kwak, MD, $\mathbf{P h D}^{2}$ \\ ${ }^{1}$ Department of Radiation Oncology, Massachusetts General Hospital, Harvard Medical School, Boston, MA; ${ }^{2}$ Department \\ of Medicine, Massachusetts General Hospital, Harvard Medical School, Boston, MA
}

In this issue of Annals of Surgical Oncology, Ejaz and colleagues report on the utilization rate and impact of postoperative chemoradiation as compared with perioperative chemotherapy alone in patients identified in the US Gastric Cancer Collaborative database. In this dataset of patients treated in the United States, the authors conclude, after propensity score matching, that patients receiving chemoradiation have improved overall survival compared with patients receiving chemotherapy only.

This analysis was performed because multiple standards of care exist for adjuvant therapy in resected, locally advanced gastric cancer. The development of both chemoradiation and chemotherapy-alone adjuvant approaches highlights the two competing risks of recurrence in patients with locally advanced gastric cancer: locoregional recurrence and metastatic recurrence. In the United States, the high rates of locoregional failure observed after surgery alone led to the design of Intergroup 0116, in which patients with margin-negative gastrectomy were randomized to observation or postoperative chemoradiation with 5-fluorouracil (5-FU), sandwiched between three cycles of Mayo Clinic schedule 5-FU. ${ }^{1}$ This study demonstrated a survival benefit of approximately $10 \%$ directly related to an improvement in locoregional control, but no difference in rates of distant metastases. In spite of these results, significant skepticism remained regarding the impact of this trial outside the United States, because more than half the patients had an insufficient, D0, lymphadenectomy. In Europe, the MAGIC study evaluated perioperative

(C) Society of Surgical Oncology 2014

First Received: 23 April 2014;

Published Online: 20 May 2014

T. S. Hong, MD

e-mail: tshong1@partners.org chemotherapy with epirubicin, cisplatin, and 5-FU versus surgery alone. ${ }^{2}$ Patients receiving chemotherapy received three cycles before and after surgery. Similarly, this study demonstrated a survival benefit of approximately $10 \%$, although this time because of a substantial improvement in distant metastases.

This current analysis by Ejaz et al. focuses on a retrospective, nonrandomized comparison of the adoption of these two Western regimens in the United States. When a group of patients treated at academic centers was analyzed, patients receiving chemoradiation appeared to have an improved overall survival in comparison to those receiving chemotherapy only, even after propensity score matching. Interestingly, the benefit was even more pronounced in patients with positive lymph nodes.

This study highlights the uncertainty that exists as to whether radiation is needed in the management of gastric cancer. Although both of these trials would suggest that surgery alone is not sufficient in locally advanced disease, adjuvant chemoradiation can be difficult to tolerate because of the large field necessary to adequately encompass at-risk nodal regions. To evaluate the role of radiation in patients receiving chemotherapy after a D2 lymph node dissection, the Adjuvant Chemoradiation Therapy in Stomach Cancer (ARTIST ) trial randomized patients between capecitabine and cisplatin (XP) for 6 cycles versus XP for 2 cycles, followed by chemoradiation with capecitabine to $45 \mathrm{~Gy}$, followed by XP for 2 more cycles (XP/XRT/XP). ${ }^{3}$ A total of 458 patients were randomized between the two arms. Patients were generally high risk, with $>85 \%$ enrolled having node-positive disease. More than half the patients had diffuse-type disease, as opposed to intestinal type. As expected from this high-volume surgical center, all patients had a D2 dissection, with a median of 40 lymph nodes evaluated in both arms. With a median follow-up of 53.2 months, the 3-year disease-free survival (DFS) was $78.2 \%$ 
TABLE 1 Outcomes of adjuvant therapy trials for resected locally advanced esophagogastric cancer

\begin{tabular}{lllll}
\hline Study & $\begin{array}{l}\text { East vs. } \\
\text { West }\end{array}$ & Intervention & $\begin{array}{l}\text { 3-Year DFS } \\
\text { surgery alone }\end{array}$ & $\begin{array}{l}\text { 3-Year DFS } \\
\text { treatment }\end{array}$ \\
\hline ACTS-GC $^{4}$ & East & S-1 & $59 \%$ & $72 \%$ \\
CLASSIC $^{5}$ & East & CapOx & $59 \%$ & $74 \%$ \\
INT0116 $^{1}$ & West & ChemoRT & $31 \%$ & $48 \%$ \\
MAGIC $^{2}$ & West & Periop ECF & $28 \%{ }^{\text {a }}$ & $38 \%^{\mathrm{a}}$ \\
\hline
\end{tabular}

ECF epirubicin, cisplatin, and 5-FU

a Estimated from Kaplan-Meier curves

in the XP/XRT/XP arm compared with $74.2 \%$ in the XP arm $(p=0.0862)$. Although this difference did not reach statistical significance, the node-positive patients appeared to derive benefit from the addition of radiation, with a 3 -year DFS of $77.5 \%$ in the XP/XRT/XP arm compared with $72.3 \%$ in the XP arm $(p=0.0365)$. Interestingly, locoregional recurrence was quite low in both arms $(4.8 \%$ in XP/XRT/XP arm vs. $8.3 \%$ in the XP arm; $p=0.3533$ ).

Although the ARTIST study would suggest that patients with node-positive disease may benefit from adjuvant chemoradiation, it remains unclear whether these results can be extrapolated to a Western population. Whether Eastern (Japan, Korean) and Western (Europe, Americas) gastric cancers represent the same disease remains an open question. When evaluating surgery-alone arms in phase III studies, the 3-year DFS in Asian studies is routinely double the rate seen in Western studies (Table 1). Some of this difference may be attributable to the more extensive lymphadenectomy generally performed in Asian countries, which could lead to both stage migration and improved local control. However, a 30\% improvement in DFS from a superior surgical technique alone seems quite remarkable. Furthermore, this difference in outcome between East and West is not restricted to the resectable setting. In the Avastin in Gastric Cancer (AVAGAST) ${ }^{6}$ phase III study evaluating the addition of bevacizumab to first-line chemotherapy in metastatic or unresectable patients, there again was a striking difference in the performance of Asian patients versus European or Pan-American patients. Asian patients benefitted least from the addition of bevacizumab. Asian patients also had the highest median survival in the chemotherapy alone arm (12.1 months, vs. 8.6 months in Europe and 6.8 months in Pan-America). Additionally,
Asian patients had the highest overall response rate with chemotherapy alone (45.5 vs. $28.2 \%$ in Europe and $36.4 \%$ in Pan-America). These observations of improved survival and response to chemotherapy in Asian patients suggest that even in the nonsurgical setting, gastric cancer in the East may be a different entity than in the West.

The current evaluation by Ejaz and colleagues is therefore important because it validates the observations of potential benefit of chemoradiation in the ARTIST trial in the United States. Similar to the ARTIST trial, the most striking benefit appears to be in node-positive patients. Although this study supports the continued use of chemoradiation in the United States, it is important to note that no substantial progress has been made in adjuvant therapy of gastric cancer since the initial publication of INT0116. Future efforts should continue to improve our understanding of the pathogenesis of gastric cancer in both the East and West, determine the optimal sequencing of therapy (preoperative vs. postoperative), and refine our patient selection for adjuvant therapy.

CONFLICTS OF INTEREST No conflicts of interest with topics under discussion

\section{REFERENCES}

1. Macdonald JS, Smalley SR, Benedetti J, et al. Chemoradiotherapy after surgery compared with surgery alone adenocarcinoma of the stomach or gastroesophageal junction. $N$ Engl $J$ Med. 2001;345:725-30.

2. Cunningham D, Allum WH, Stenning SP, et al. Perioperative chemotherapy versus surgery alone for resectable gastroesophageal cancer. N Engl J Med. 2006;355:11-20.

3. Lee J, Lim DH, Park SH, et al. Phase III trial comparing capecitabine plus cisplatin versus capecitabine plus cisplatin with concurrent capecitabine radiotherapy in completely resected gastric cancer with D2 lymph node dissection: the ARTIST trial. J Clin Oncol. 2011;30:268-73.

4. Sakuramoto S, Sasako M, Yamaguchi T, et al. Adjuvant chemotherapy for gastric cancer with $\mathrm{S}-1$, an oral fluoropyrimidine. $N$ Engl J Med. 2007;357:1810-20.

5. Bang Y, Kim Y, Yang H, et al. Adjuvant capecitabine and oxaliplatin for gastric cancer after D2 gastrectomy (CLASSIC): a phase 3 open-label randomised controlled trial. Lancet. 2012;379:315-21.

6. Ohtsu A, Shah MA, Van Cutsem E, et al. Bevacizumab in combination with chemotherapy as first-line therapy in advanced gastric cancer: a randomized, double-blind, placebo-controlled phase III study. J Clin Oncol. 2011;29:3986-76. 